\title{
ELECTROHYDRODYNAMIC JET PRINTING: A 3D PRINTING TECHNIQUE FOR SENSOR FABRICATION
}

\author{
C.P. Pannier ${ }^{*}$, Z. Wang ${ }^{2}$, D.J. Hoelzle ${ }^{2}$ and K.L. Barton ${ }^{1}$ \\ ${ }^{1}$ University of Michigan, Ann Arbor, Michigan, USA \\ ${ }^{2}$ University of Notre Dame, Notre Dame, Indiana, USA
}

\begin{abstract}
Electrohydrodynamic jet (e-jet, EHD) printing is a candidate technique for maskless MEMS fabrication. It achieves improved spatial resolution compared to inkjet printing, and has a wider range of acceptable inks. This paper presents new results for high viscosity ink, sub $2 \mu \mathrm{m}$ polymer features, and printed electrodes. We demonstrate submicron precision in droplet-to-droplet positioning with in situ monitoring. Rapid, sub-optical measurement of printed patterns provides a unique method for characterizing e-jet printing performance with specific interest in the applications of liquid resist patterning for nanoimprint lithography and the direct printing of thermally-sinterable conductive lines for sensor and actuator fabrication. Directions for the development of e-jet printing are discussed.
\end{abstract}

\section{INTRODUCTION}

Current manufacturing processes for micro and nanotechnology are inherently expensive due to required cleanliness, precision motion control, custom photomasks, and instrumentation. Large volume production is required to recover capital expenses. The recent extension of the maskless additive manufacturing (AM) paradigm to the microscale relaxes substrate planarity restrictions, eliminates harsh chemical and high temperature processes, and reduces material consumption [1]. Hence micro-additive manufacturing ( $\mu$-AM) enables prototype or low volume production at the microscale like mesoscale AM does at the mesoscale.

Electrohydrodynamic jet printing (e-jet, EHD) is one such $\mu$ AM approach in controlled ink droplet formation and deposition through the generation of an electric field between a charged micro-capillary pipette and a grounded substrate as shown in Fig. 1 [1]. In recent years, e-jet printing has demonstrated superior resolution to inkjet printing at a lower cost than lithographic fabrication techniques [2]. Because of the ability to costeffectively print high-resolution droplets and lines with a range of inks, e-jet printing has shown tremendous promise for applications such as printing metallic $(\mathrm{Ag})$ interconnects for printed electronics [3], [4], [5] and bio-sensors [6], [7], [8].

This paper describes the e-jet printing process, its capabilities and limits, and then compares e-jet to inkjet printing for $\mu-\mathrm{AM}$, and presents new high-resolution printed patterns on an integrated e-jet printer and scanner.

\section{BACKGROUND: E-JET PRINTING Applications}

High-resolution liquid deposition is needed for microfabrication, photoresist patterning, and functionalizing microsystems. E-jet printing has emerged as a cost effective and versatile, high-resolution AM technique for meeting this highresolution liquid deposition need. E-jet applications include: 1) prototyping microdevice designs, 2) depositing resist droplets such as for nanoimprint lithography, 3) direct fabrication of customizable microsystems, 4) multi-material and heterogeneous fabrication utilizing parallel deposition capabilities, and 5) selective deposition by drop-on-demand that enables targeted functionality.
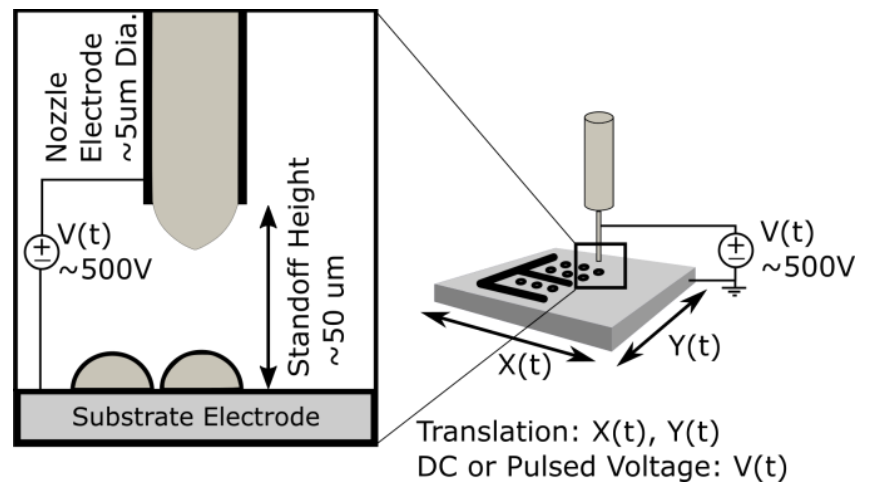

Figure 1: Schematic of the e-jet printing setup. Control variables include offset height and applied voltage. Increased voltage leads to a Taylor cone formation and droplet ejection from the cone tip to print droplets or continuous lines.

E-jet printed sensor applications are reviewed in [1] including gas, temperature, chemical analyte sensing. A route to producing MEMS ceramic sensors is proposed to use e-jet printed viscous polymer precursors to ceramics [9]. E-jet printed biomolecules on photonic crystals have been shown to increase sensitivity to biomarkers [10]. Recent e-jet printed sensors include the electrodes and photoconductive material for a photoresistor [11], and semiconducting $\mathrm{SnO}_{2}$ lines (5 $\mu \mathrm{m}$ width) to sense $\mathrm{NO}$ gas [12].

One potential application to utilize the high-resolution and precision printing capabilities of e-jet printing is depositing resist before UV nanoimprint lithography (UV-NIL). Standard UV-NIL approaches use a spin-coated layer or inkjet printed pattern of resist on a substrate. An imprint mold with $\sim 10 \mathrm{~nm}$ scale features is pressed onto the resist before UV exposure. Printed resist patterns offer the advantage over films for faster filling of the mold and air bubble dissolution [13]. E-jet-printing smaller resist droplets could further improve UV-NIL performance.

\section{Process}

An e-jet printer consists of a hydrophobic, conductive microcapillary nozzle that undergoes an applied voltage relative to a counter electrode at or below the substrate. Under this applied voltage, the ink meniscus forms a cone that extends into a narrow jet to deliver liquid to the substrate. The jetting behavior is affected by nozzle geometry, standoff height between the nozzle and the substrate, and material surface tension. Droplet base diameter $\left(d_{\text {base }}\right)$ scales with nozzle inner diameter $\left(d_{n}\right)$, and inversely with applied voltage $(V)$ as given in Eq. (1) [14], [15].

$$
d_{\text {base }} \propto d_{n}^{m} V^{-p}, \quad 0<m<1, \quad 0<p<1
$$

For a nozzle diameter of $d_{n}=2 \mu \mathrm{m}$, applied voltages of $V=$ 200 to $800 \mathrm{~V}$ are typical to achieve base diameter values smaller than the nozzle diameter, $d_{\text {base }}<2 \mu \mathrm{m}$. Continuous and pulsed modes of applied voltage for e-jet printing are shown in Fig. 2. Pulsed printing enables controlled jetting at a specified time or 


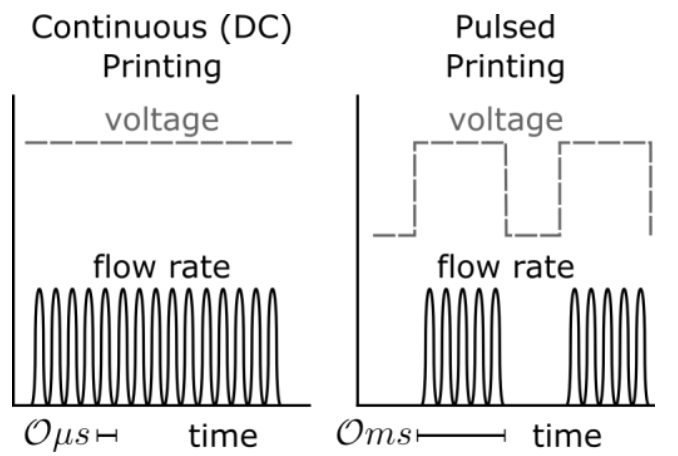

Figure 2: Schematic of continuous and pulsed printing. Nonrectangular pulse waveforms including sinusoids are also used.

position (for a nozzle voltage signal that is coordinated with substrate motion) with a specific volume determined by the pulse width [16].

\section{Capabilities}

Diverse materials have been e-jet printed, as reviewed in [1]. Conductive traces have been deposited by printing and then sintering metal nanoparticle suspensions. Biological materials and UV-curable polymers have also been patterned by e-jet printing. E-jet printed droplets and lines carry an electrical charge from the deposition process, thus e-jet printed charges can be patterned on a substrate for self-assembly [5].

\section{Limitations}

The standoff height affects the electric field strength at the meniscus and thus the jetting behavior. A typical standoff height for sub- $10 \mu \mathrm{m}$ printed features is $<50 \mu \mathrm{m}$. A substrate height variation of $\pm 10 \mu \mathrm{m}$ can inhibit ink ejection or allow undesirable jetting behavior such as satellite droplets or scattering. The sensitivity to substrate height variations has inspired research into e-jet sensing and control as well as printhead design [17], [18], [19]. In addition to standoff height sensitivity, EHD hysteresis of the meniscus during jetting can cause the first droplets of a pattern to be larger or smaller than subsequent droplets. Additionally, nozzle clogging is a concern for e-jet printing, especially with small nozzle openings of $0.1-10 \mu \mathrm{m}$. Ink suspensions must be filtered to prevent clogging and volatile solvents are avoided.

\section{Comparison with Inkjet Printing}

Drop-on-demand inkjet printing has been used to print electrical, optical and biological materials and 3D structures [20]. Either thermal bubble or piezoelectric actuation may be used, but thermal actuation restricts ink formulation to a limited boiling point range. Piezo inkjet (PIJ) ink choice is restricted by the requirement of an ink viscosity in the range of about 2 to $50 \mathrm{mPa}$-s. The temperature dependence of liquid viscosity allows some viscous inks to be PIJ printed at elevated temperatures [20]. The complex ink ejection mechanism requires MEMS-fabricated multithousand nozzle printheads with engineered features to address clogging, satellite droplet, and nozzle life issues [20].

E-jet printing provides several orders of magnitude advantage ( $<100 \mathrm{~nm}$ droplets) over the smallest achievable inkjet droplet size $(>20 \mu \mathrm{m})$, which drives feature resolution. Assuming droplets form spherical caps with fixed contact angle, the base diameter is related to contact angle and droplet volume as shown in Fig. 3. Ejet printing has achieved sub $100 \mathrm{~nm}$ feature sizes while printing onto unstructured substrates with a variety of materials [1], [6]. The ability of nearfield e-jet to produce orders of magnitude smaller droplet volumes gives it nearly three orders of magnitude advantage in smallest droplet base diameter.

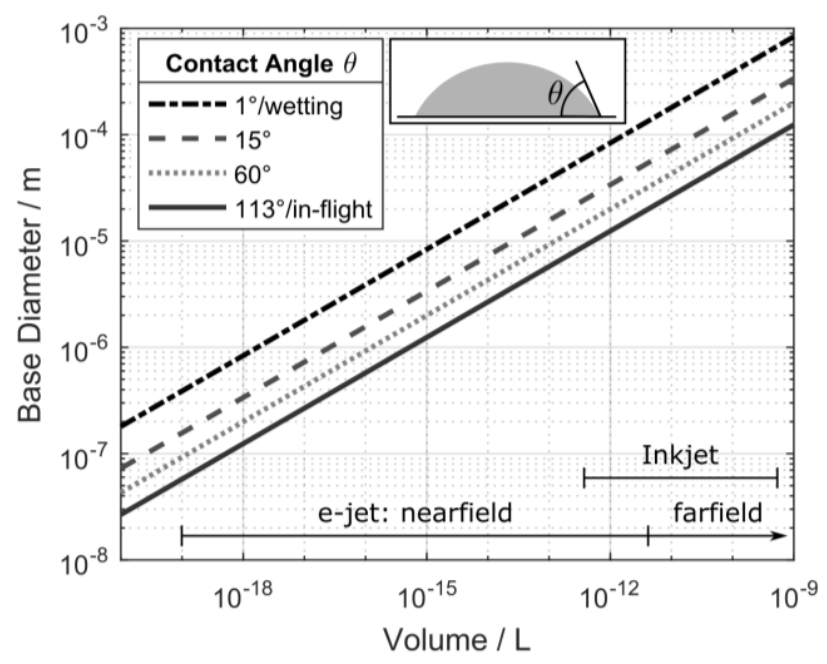

Figure 3: Droplet volumes and base diameters are related in the spherical cap droplet model for various equilibrium contact angles, $\theta$. The $1^{\circ}$ contact angle shows that highly wetting systems have a base diameter 5 to 10 times the in-flight diameter. Typical PIJ droplet volumes are 0.5-500 pL [20].

\section{METHODS}

E-jet printing was performed on a custom printing and scanning testbed at the University of Michigan, shown in Fig. 4. This printer has linear motor $\mathrm{X}, \mathrm{Y}$ and $\mathrm{Z}$ stages with bidirectional repeatability $\sim 0.2 \mu \mathrm{m}$. The substrate plane is aligned with the staging plane using a manual tip/tilt stage. A UV light source is used to cure printed patterns. The setup also contains an atomic force microscope (Nanite AFM) for in situ sub-optical measurement of printed, cured patterns. The experimental setup is automated for printing, curing, and scanning of arrays of patterns. The vacuum substrate mount can accommodate up to $100 \mathrm{~mm}$ wafer substrates. For the droplet printing examples shown, pulsed e-jet printing was performed with time delays before and after substrate motion.

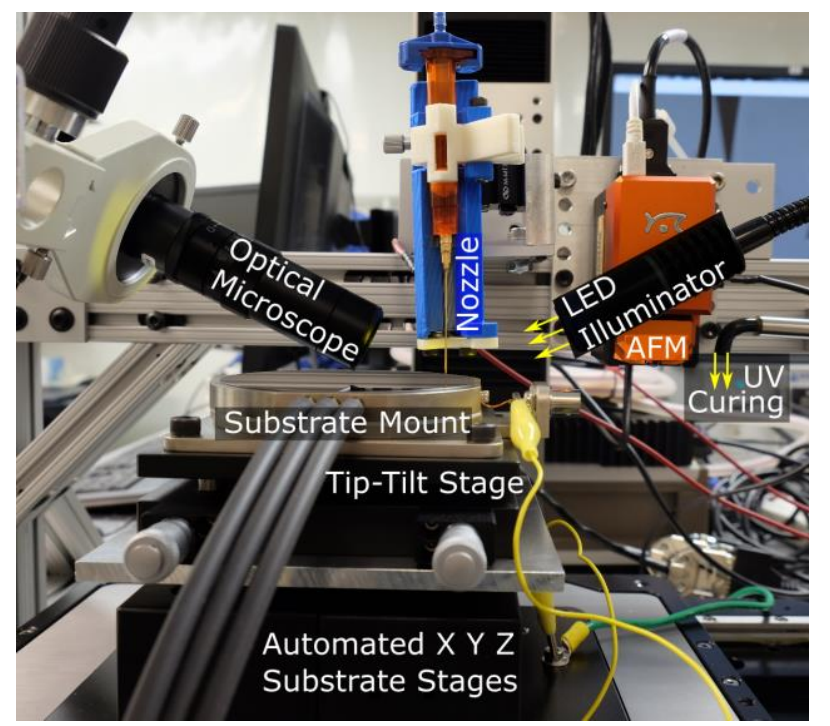

Figure 4: The printer components are labeled with stations for the nozzle, AFM scanner and UV curing light. 

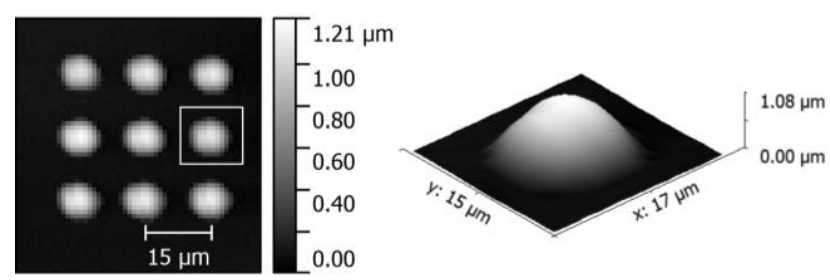

Figure 5: AFM scan and $3 D$ view of boxed droplet from a $3 \times 3$ field of e-jet printed high-viscosity droplets $(17,500 \mathrm{mPa}-\mathrm{s})$ : mean diameter $11 \mu \mathrm{m}$, mean volume $47 \mathrm{fL}$. Printed features are UVcured Loctite 3526 Light Cure Acrylic. Raw AFM scan base flattened.

\section{RESULTS}

A high viscosity $(17,500 \mathrm{mPa}$-s) UV-curable acrylic adhesive, Loctite 3526, was successfully e-jet printed at room temperature. Fig. 5 shows one such pattern with a droplet diameter of $11 \mu \mathrm{m}$. This viscosity is nearly three orders of magnitude higher than the viscosity of inks that can be inkjet printed. The e-jet printing of this high viscosity ink exhibited an electrospinning behavior within the short $(45 \mu \mathrm{m})$ distance of ink travel. Pulsed printing was used to limit jet whipping to achieve individual droplets.

A UV-curable mercapto ester mixture, Norland Optical Adhesive 81 (NOA81), of viscosity $300 \mathrm{mPa}$-s, was e-jet printed with decreasing pitch to find the minimum pitch for a given set of printing parameters in Fig. 6. The onset of merger at a pitch of 12 $\mu \mathrm{m}$ indicates $d_{\text {base }}+$ scatter $<13 \mu \mathrm{m}$ for these printing parameters.

A high resolution $(<10 \mu \mathrm{m})$ pattern was e-jet printed in Fig. 7 with two passes of equal density, with the second pass printed in the interstices of the first pass. Droplet positioning in the second pass showed scattering from its intended print site, possibly due to forces from the first printed and cured pass droplets.

A high resolution pattern with $2 \mu \mathrm{m}$ pitch was e-jet printed in one pass in Fig. 8 to leave an uncovered area with the text "EJET." This pattern highlights the ability to print high-resolution images with microscale precision and accuracy in droplet placement.

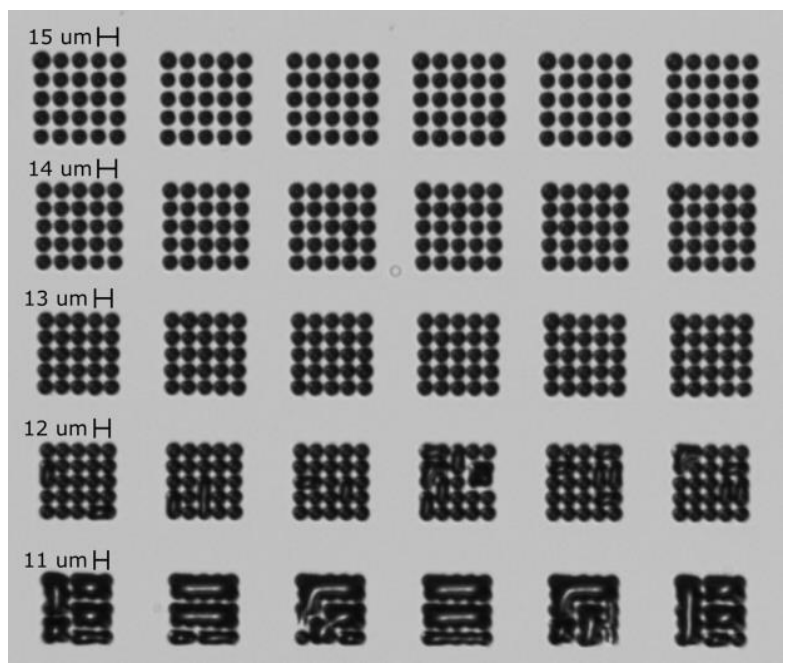

Figure 6: A micrograph of an e-jet printed test pattern in which the printed pitch decreases by $1 \mu \mathrm{m}$ in each row until merger occurs. Ink is NOA81 printed on a gold-coated glass slide. In this test, merger begins at a pitch of $12 \mu \mathrm{m}$.

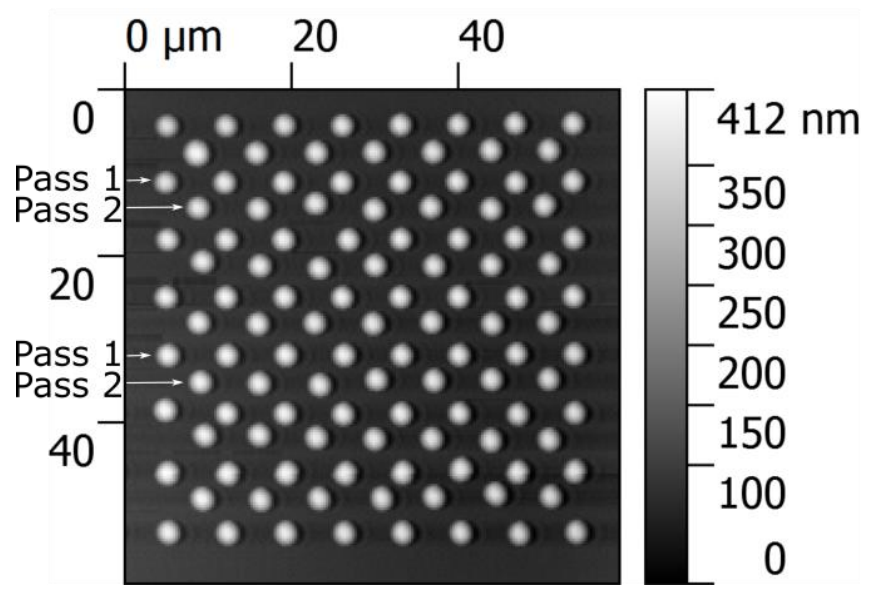

Figure 7: AFM scan of an e-jet printed field: mean diameter 3.4 $\mu \mathrm{m}$, mean volume $1.2 \mathrm{fL}$. Pattern printed in two passes: one pass of $8 \times 8=64$ droplets and a second pass of $7 \times 7=49$ droplets. Droplets were printed at $X$ and $Y$ pitches of $5 * \operatorname{sqrt}(2)=7.1 \mu \mathrm{m}$, giving a designed diagonal pitch of $5 \mu \mathrm{m}$ between droplets of different passes. Printed features are UV-cured NOA81. Raw AFM scan base flattened and processed for step line removal.

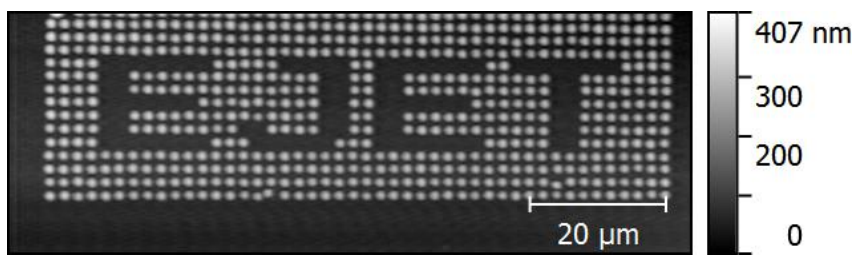

Figure 8: Atomic force microscope (AFM) scan of a field of $15 \times 46$ e-jet printed droplets: mean diameter $1.6 \mu \mathrm{m}$, mean volume $0.3 \mathrm{fL}$. Material is UV-cured NOA81. Raw AFM scan base flattened.

To cover an area of $1 \mathrm{~mm}^{2}$ with a droplet array of similar quality to Fig. 8 using the single-nozzle e-jet printer in Fig. 4 requires 7 minutes with 10,000 droplets in pulsed printing $(\sim 15$ $\mathrm{pL} / \mathrm{min}$ time-averaged flow rate). In continuous e-jet printing, the same $1 \mathrm{~mm}^{2}$ area may be covered in $<1$ minute with parallel lines of $10 \mu \mathrm{m}$ pitch. These reported e-jet speeds are not yet optimized for commercial applications. E-jet printing throughput can be improved at the cost of reduced spatial resolution by using a larger nozzle at higher voltage (farfield e-jet printing). This might be a useful method of depositing, at inkjet resolution, highly viscous inks that cannot be inkjet printed.

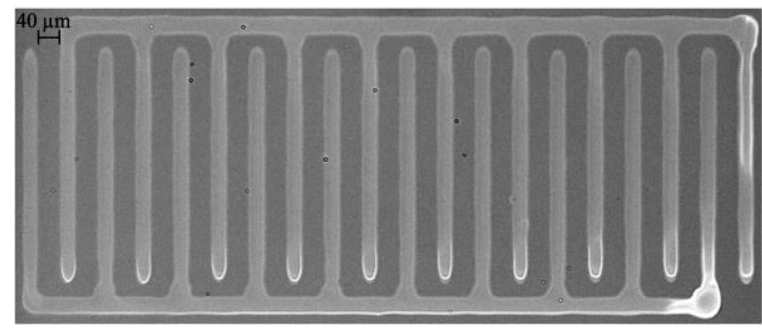

Figure 9: A micrograph of a silver interdigitated electrode with $500 \mu \mathrm{m}$ length and $30 \mu \mathrm{m}$ line widths, printed in 10 seconds. 
Figure 9 shows an e-jet printed interdigitated electrode pattern. The ink is a $25 \mathrm{wt} . \%$ silver nanoparticle solution in an organic solvent (UT Dots, Inc.). The highly wetting ink spreads to $30 \mu \mathrm{m}$ widths and exhibits the coffee ring effect. The deposition of continuous lines was performed at speeds up to $3.5 \mathrm{~mm} / \mathrm{s}$. Repeated patterns of the e-jet prints shown in Fig. 5-9 are candidates for implementing the advanced monitoring and control strategies recently presented [21].

\section{CONCLUSION}

The presented capabilities and new prints of insulating resists and silver nanoparticle solutions has shown that e-jet printing is an alternative micro-fabrication technique for: 1) prototyping microdevice designs, 2) depositing resist droplets for nanoimprint lithography, 3) direct fabrication of customizable microsystems, 4) multi-material and heterogeneous fabrication utilizing parallel deposition capabilities, and 5) selective deposition through dropon-demand that enables targeted functionality. Despite these advantages, there remain unexplored challenges in ink and surface chemistry characterization, printhead design, and integrated control to further enhance capabilities for printing functional devices. Future work will explore novel approaches for addressing these challenges.

\section{ACKNOWLEDGEMENTS}

This work was supported in part by NSF Awards CMMI1434660, CMMI-1434693, GRFP-1256260, and start-up funds from the University of Notre Dame. Travel support has been generously provided by the Transducer Research Foundation.

\section{REFERENCES}

[1] M.S. Onses, E. Sutanto, P.M. Ferreira, A.G. Alleyne, and J.A. Rogers, "Mechanisms, Capabilities, and Applications of High-Resolution Electrohydrodynamic Jet Printing," Small, 11, (2015).

[2] K.L. Barton, S. Mishra, K.A. Shorter, A.G. Alleyne, P. Ferreira, and J.A. Rogers, "A desktop electrohydrodynamic jet printing system," Mechatronics, 20, (2010).

[3] D-H. Youn, S-H. Kim, Y-S. Yang, S-C. Lim, S-J. Kim, S-H. Ahn, H-S Sim, S-M Ryu, D-W Shin, and J-B Yoo, "Electrohydrodynamic micropatterning of silver ink using near-field electrohydrodynamic jet printing with tilted-outlet nozzle," App. Phys. A, 96, 4, (2009).

[4] D-Y. Lee, J-C. Lee, Y-S Shin, S-E Park, T-U Yu, Y-J Kim, and J Hwang, "Structuring of conductive silver line by electrohydrodynamic jet printing and its electrical characterization,” J. Phys: Conf. Ser., 142, 012039, (2008).

[5] J-U. Park, S. Lee, S. Unarunotai, Y. Sun, S. Dunham, T. Song, P.M. Ferreira, A.G. Alleyne, U. Paik, and J.A. Rogers, "Nanoscale, Electrified Liquid Jets for High-Resolution Printing of Charge," Nano Letters, 10, 2, (2010).

[6] J-U. Park, J-H Lee, U. Paik, Y. Lu, and J.A. Rogers, "Nanoscale Patterns of Oligonucleotides Formed by Electrohydrodynamic Jet Printing with Applications in Biosensing and Nanomaterials Assembly," Nano Lett., 8, 12, (2008).

[7] S. Jayasinghe, A. Qureshi, and P. Eagles, "Electrohydrodynamic Jet Processing: An Advanced ElectricField-Driven Jetting Phenomenon for Processing Living Cells," Small, 2, 2, (2006).

[8] K. Kim, B. Lee, G. Hwang, J.H. Lee, and S. Kim, "Drop-ondemand patterning of bacterial cells using pulsed jet electrospraying," Anal. Chem., 82, 5, (2010).

[9] H. Duan, C. Li, W. Yang, B. Lojewski, L. An, and W. Deng,
"Near-field electrospray microprinting of polymer-derived ceramics," J. Microelectromechanical Syst., 22, 1, (2013).

[10] S. George, V. Chaudhery, M. Lu, M. Takagi, N. Amro, A. Pokhriyal, Y. Tan, P. Ferreira, and B. T. Cunningham, "Sensitive detection of protein and miRNA cancer biomarkers using silicon-based photonic crystals and a resonance coupling laser scanning platform.," Lab Chip, 13, 20, (2013).

[11] S. Ali, J. Bae, C.H. Lee, K.H. Choi, and Y.H. Doh, "Flexible and passive photo sensor based on perylene/graphene composite," Sensors Actuators, B Chem., vol. 220, (2015).

[12] C-Y. Kim, H. Jung, H. Choi, and D. Choi, "Synthesis of onedimensional $\mathrm{SnO} 2$ lines by using electrohydrodynamic jet printing for a NO gas sensor," J. Korean Phys. Soc., 68, 2, (2016).

[13] K. Usuki, S. Wakamatsu, T. Oomatsu, K. Kodama, and K. Kodama, "Approaches to rapid resist spreading on dispensing based UV-NIL," Proc. of SPIE, 7970, (2011).

[14] C. H. Chen, D.A. Seville, and I.A. Aksay, "Scaling laws for pulsed electrohydrodynamic drop formation," Appl. Phys. Lett., 89, 12, (2006).

[15] H. K. Choi, J.-U. Park, O. O. Park, P. M. Ferreira, J. G. Georgiadis, and J.A. Rogers, "Scaling laws for jet pulsations associated with high-resolution electrohydrodynamic printing," Appl. Phys. Lett., 92, 12, (2008).

[16] S. Mishra, K. L. Barton, a G. Alleyne, P. M. Ferreira, and J. a Rogers, "High-speed and drop-on-demand printing with a pulsed electrohydrodynamic jet," J. Micromechanics Microengineering, 20, 9, (2010).

[17] L. Tse and K. Barton, "Airflow assisted printhead for highresolution electrohydrodynamic jet printing onto nonconductive and tilted surfaces," Appl. Phys. Lett., 107, 5, (2015).

[18] B. Altin, L. Tse, and K.L. Barton, "Visual feedback based droplet size regulation in electrohydrodynamic jet printing," Proc. ASME DSCC2014, San Antonio, TX, 10/22-24/14, ASME, (2014), pp. 6110.

[19] D. Hoelzle, K. Barton, "On Spatial Iterative Learning Control via 2-D Convolution: Stability Analysis and Computational Efficiency", Trans. Control Sys. Tech., Accepted, 2015.

[20] S.D. Hoath, ed., Fundamentals of Inkjet Printing: The Science of Inkjet and Droplets, Wiley-VCH, Weinheim, Ger., 2016.

[21] Z. Wang, C.P. Pannier, L. Ojeda, K.L. Barton, and D.J. Hoelzle, "An Application of Spatial Iterative Learning Control to Micro-Additive Manufacturing," Proc. ACC 2016, July 6-8, 2016.

\section{CONTACT}

*C.P. Pannier, PANNIER@UMICH. edu 